\title{
Glassy Polymers as Matrices for Advanced Composites
}

\author{
Eduard F. OLEINIK \\ Institute of Chemical Physics, USSR Academy of Sciences, \\ Kosygin str. 4, Moscow, 117334, USSR
}

(Received August 18, 1986)

\begin{abstract}
This paper is concerned with several fundamental aspects of the glassy polymer structure and properties. One aspect is chemical vitrification, the process whereby the reactants are transformed from the liquid into the glassy state due to chemical reactions. The influence of that transformation on the Young moduli and $T_{\mathrm{g}}$ of formed polymers is considered. This is exemplified by the epoxy cure process. Another aspect is the mechanism of plastic deformation of polymeric glasses and the third one is the isothermal enthalpy and volume relaxations in glassy polymers during physical aging. Choice of these topics is connected with their importance for polymer materials and advanced composite science. Some new experimental results for glassy polymers are introduced. The plastic deformation of glassy polymers is interpreted in the terms of two independent deformation modes. Mode I (low deformation temperature mode) is crystal-like and based on the nucleation and growth under stress of defects which have many features of dislocations. The second (high deformation temperature) Mode II is the diffusion motion of vacancies (holes) due to simultaneous action of stress and temperature. In the simultaneous measurement of volume and enthalpy isothermal relaxation a new type of relaxation asymmetry for sample contraction and expansion was found.

KEY WORDS Glassy-Polymers / Vitrification / Cure / Plastic Deformation / Enthalpy-Volume Recovery / Dimension Recoverability / DislocationLike Defects /
\end{abstract}

During the last decade interest in the glassy state of matter has sharply increased. The reasons are both technological and scientific. Many new glassy substances have achieved a pronounced importance as they became modern advanced technical materials such as metallic glasses, amorphous semiconductors and glassy polymers. ${ }^{1-3}$ Growing interest of material scientists and solid state physicists in amorphous structure became evident ${ }^{4,5}$ also.

Polymer science and technology are playing a remarkable role in this technical and scientific trend.

From the scientific point of view polymers have introduced a new type of structure in the field of the amorphous state due to their inherent noncrystallizability. That property of polymers is the function of configurational disorder along macromolecular chains. It has opened new challenging problems in modern physics, thermodynamics and physical chemistry $^{6}$ - the problem of the equilibrium structures for amorphous solids and any solids in general.

The role of the polymer glassy state in research and development of advanced composites is very important. Practically, polymer matrices in a major portion of useful advanced composites are glassy polymers ${ }^{7}$ and advanced composites with polymer matricies are now broadly utilized by different industries. Modern demands for new heat resistant, rigid, strong and ductile materials are requiring polymer scientists to create new polymers with improved characteristics. And these demands are turning the interests of polymer com- 
munity to new glassy polymers and the processes of their formation more and more.

However the scientific understanding of the structure-property relationship of glassy polymers (and the glassy state of matter as well) is now at a level which is not satisfactory. In most cases this deficit of knowledge is a limiting factor in the development of better polymer materials.

The aim of this short introduction is only to emphasize the importance of deep fundamental researches in polymer material science and glassy state of polymers as well.

There are, of course, thousands of factors influencing end-use properties of glassy polymer either as the material or a part of it. This paper will be only concerned with three aspects of the problem which seem quite important and general from the material science point of view. The choice of the topics is quite personal and does not diminish the role of other approaches.

These three topics are the following:

i) Chemical vitrification: the process during which the reacting system is transformed from the liquid to the glassy state due to isothermal chemical reaction;

ii) Plastic deformation of glassy polymers;

iii) Enthalpy and volume relaxation of polymer glasses during physical aging.

\section{CHEMICAL VITRIFICATION}

Chemical vitrification or solidification is a process in which some system of high fluidity is transformed into a rigid glassy matter of very low fluidity due to molecular weight growth during a chemical reaction. Polymer chemists are well acquainted with the phenomenon through well known processes such as the cure of thermosets, formation of adhesive joints and bulk polymerization of some monomers (styrene, etc).

The simplest case of chemical vitrification is isothermal polymerization of styrene in bulk. Liquid monomer during isothermal polymeri- zation is converted to the glassy atactic polymer. The main reason for this conversion is a higher packing density of macromolecules in comparison with the monomer packing density at polymerization temperature.

In the styrene-PS transformation chemical vitrification is only a physical process and the glassy state of the polymer becomes preferable because the amount of free volume in the reacting system reaches some critical value due to the change of reactant molecular structure. The situation becomes more complex in thermosetting reactions of networks formation, e.g., curing of multifunctional epoxy..$^{8-10}$

In this case two different processes coexist. One is the formation of a gel-the infinite size molecular network. ${ }^{89}$ Another is vitrification of a reacting mixture due to the increase of packing density.

The reaction conversion at gel-point does not depend on cure temperature $T_{\text {cure }}$, however conversion at which chemical vitrification starts and proceeds strongly depends on $T_{\text {cure }}$. At comparatively high $T_{\text {cure }}$ (at $T_{\text {cure }}>T_{\mathrm{g}}^{\max }$ - the glass transition temperature of a completely cured network) a reacting system does not vitrify into the glassy state even at complete cure. ${ }^{10}$ At low $T_{\text {cure }}$ a reacting system does not reach the gel-point conversion due to vitrification at lower conversions. To clarify possible cases it is convenient to use the so called TTT (Time-Temperature-Transformation) diagramms. ${ }^{8}$ The kinetics of chemical reactions with the complication of diffusion control in the vicinity of chemical vitrification is well documented. ${ }^{8,9}$ However, one important aspect of the phenomenon namely the influence of chemical vitrification on the properties and structure of the formed polymer are not investigated sufficiently. In this paper I will try to demonstrate this influence by an example of the cure reaction of difunctional epoxy ethers with aromatic amines.

This simple system shows the quite pronounced influence of the chemical vitrification 


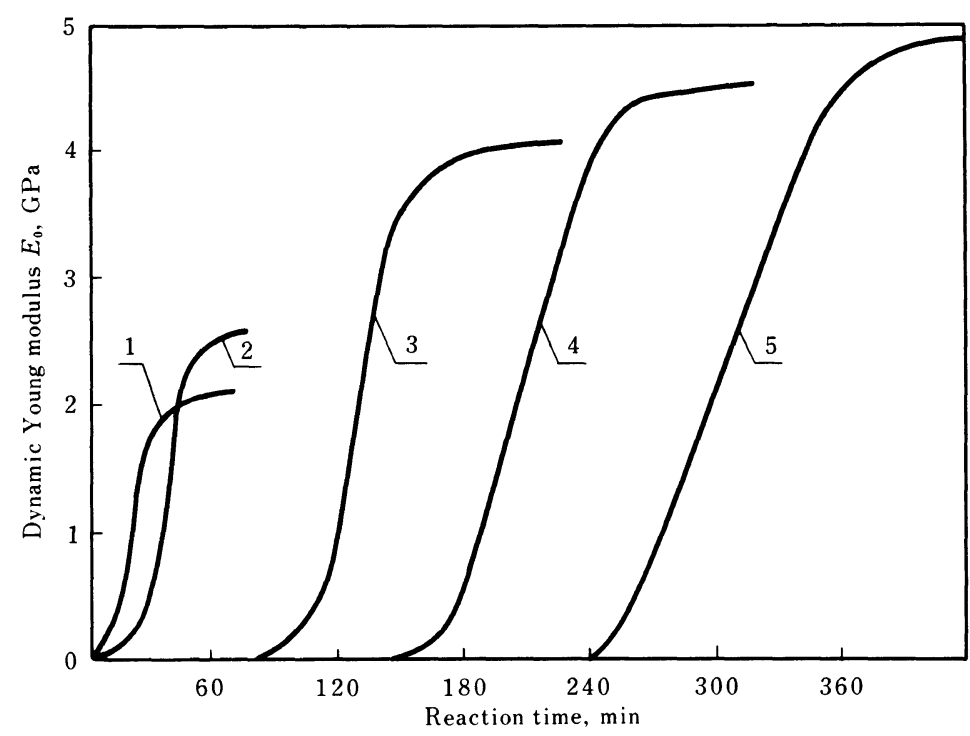

Figure 1. Change of the dynamic Young modulus $(2-10 \mathrm{~Hz})$ of DGER- $m$-PhDA $(P=1)$ mixture during isothermal cure at different $T_{\text {cure }} . T_{\text {cure }}: 1,120^{\circ} \mathrm{C} ; 2,100^{\circ} \mathrm{C} ; 3,70^{\circ} \mathrm{C} ; 4,60^{\circ} \mathrm{C} ; 5,50^{\circ} \mathrm{C} . \alpha_{\text {dif }}: 1,0.92 ; 2,0.90 ; 3$, $0.87 ; 4,0.84 ; 5,0.8$.

rate on some mechanical and thermal properties of the cured polymer. ${ }^{10}$ And this example is interesting because polymer matrices in modern advanced composites are based mainly on epoxy networks. ${ }^{7,11}$

Figure 1 shows the influence of chemical vitrification on the Young moduli of a reacting epoxy-amine mixture. All curves reflect the time behaviour of the same chemical systemdiglycidyl ether of resorcinol (DGER) mixed with $m$-phenylenediamine ( $m$-PhDA) with amine/epoxy groups molar ratio $P=$ $\mathrm{NH} / \mathrm{EP}=1$ in isothermal cure at different $T_{\text {cure }}$ $\left(50-120^{\circ} \mathrm{C}\right)$.

The cure reaction

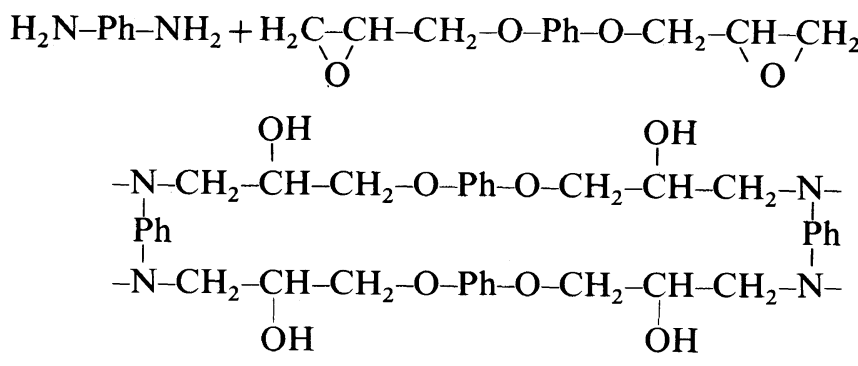

was carried out up to the diffusional limit of conversion $^{10} \alpha_{\text {dif }}$ at each $T_{\text {cure }}$. During cure under chosen conditions the reacting system becomes more and more viscous, than passes through the gel-point, and then vitrifies into the glassy state where the thermosetting proc- ess practically stops. Final conversion $\alpha_{\text {dif }}$ for given $T_{\text {cure }}$ are in the range of magnitudes $\alpha_{\text {dif }}=0.8-0.92$ that is all formed networks have a high concentration of chemical crosslinks. ${ }^{10}$

Stoichiometric system can not be cured to. 
$100 \%$ due to topological constraints. The maximum conversion $\alpha^{\max }$ which may be reached in this particular system due to the main thermosetting reaction (I) is $\alpha^{\max }=$ $0.92 \pm 0.01 .^{10}$

From the chemical point of view each unreacted functional group of the system $(\sim \mathrm{O}$ and $\sim \mathrm{NH}$ ) is a chemical defect in the network.

What can be concluded from Figure 1? Chemical defect concentration is decreasing with the increase of $T_{\text {cure }}$. However the Young moduli of the cured polymer is decreasing in that direction and vice versa.

The modulus increase is quite high with $T_{\text {cure }}$ decrease. The cured polymer annealing cannot change its Young modulus to that extent. ${ }^{10}$ It is evident that the reason for this modulus change is not chemical and, perhaps, has a structural origin.

From Figure 1 one may see that the higher the end modulus of cured polymer the smaller the rate of the vitrification process (slope of moduli increase). This result, perhaps, points out the importance of the structural relaxation rate in a polymer liquid before vitrification.

The property and structure of the glassy state obtained at low $T_{\text {cure }}$ can not be reached by any other way. It is evident from Figure 2 that the DGER- $m$-PhDA $(P=1)$ system initially was cured in the Dynamic Mechanical Analyser DMA-980 du Pont at $T_{\text {cure }}=50^{\circ} \mathrm{C}$ up to $\alpha_{\text {dif }}$ of this $T_{\text {cure }}$. The dynamic Young modulus was measured during the entire cure reaction. Then $T_{\text {cure }}$ was changed by jump to $T_{\text {cure }}=70^{\circ} \mathrm{C}$ and then again to $T_{\text {cure }}=100^{\circ} \mathrm{C}$. In all cases the cure time was sufficient for reaching $\alpha_{\text {dif }}$. From Figure 2 one may see that the polymer cured at low $T_{\text {cure }}$ up to the corresponding $\alpha_{\text {dif }}$ softens first of all after the jump-increase of $T_{\text {cure }}$ and then is vitrified to a new values of the $\alpha_{\text {dif }}$ and Young modulus due to the following thermosetting process. Obviously $\alpha_{\text {dif }}$ at $T_{\text {cure }}^{2}$ (and $T_{\text {cure }}^{3}$ ) is higher than $\alpha_{\text {dif }}$ at $T_{\text {cure }}^{1}\left(T_{\text {cure }}^{1}<T_{\text {cure }}^{2}\right)$.

It is quite important that the upper limit of the Young modulus decreases with each sub-

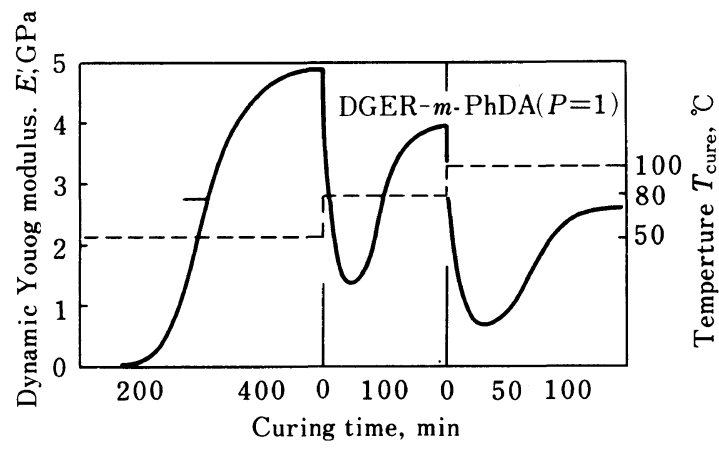

Figure 2. Change of the dynamic Young modulus of DGER- $m$-PhDA $(P=1)$ mixture during isothermal cure with consequent jump-changes of $T_{\text {cure }}$.

sequent cure step. It shows that the cured polymer "forgets" its original structure and properties at heating to $T>T_{\text {cure }}$.

Another very important physical and technical characteristic of polymeric glasses is their $T_{\mathrm{g}}$. Chemical vitrification changes $T_{\mathrm{g}}$ dramatically. First results one may see in Figure 2. Jump-increase of $T_{\text {cure }}$ is always followed by sample softenning and only then following cure reaction makes the sample rigid again.

Simple logic indicates that sample softenning should always appear when the temperature becomes a little higher than $T_{\text {cure }}$. The situation becomes clear from Figure 3 . Here are shown the time-temperature moduli changes for two samples cured at different $T_{\text {cure }}\left(T_{\text {cure }}^{1}<T_{\text {cure }}^{2}\right)$. It is evident that $T_{\mathrm{g}}$ and $T_{\text {cure }}$ should be very close to each other and the difference between them is a matter of definition. If one defines $T_{\mathrm{g}}$ as a point of the beginning of modulus drop on the $E=$ $f(T)$ curve then the relation

$$
T_{\mathrm{g}}=T_{\text {cure }}
$$

should hold.

However, if the operating definition of $T_{\mathrm{g}}$ is taken as the inflection point on $E=f(T)$ curve, as it is usually accepted, the relation is

$$
T_{\mathrm{g}}=T_{\text {cure }}+1 / 2 \Delta\left(T_{\mathrm{g}}\right)
$$

where $\Delta\left(T_{\mathrm{g}}\right)$ is the half-width of the glass transition interval. 


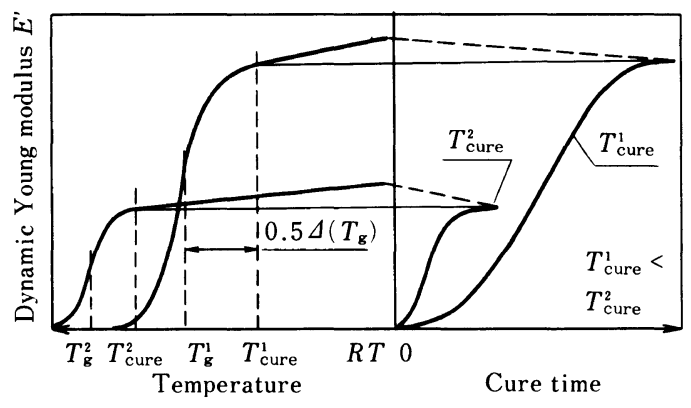

Figure 3. The scheme of correlations between $T_{\text {cure }}$ and $T_{\mathrm{g}}$.

Right side: the moduli changes in isothermal cure at two different $T_{\text {cure }}$. Dotted lines, moduli changes at cooling cured samples to room temperature.

Left side: the moduli changes of cured samples on heating.

Experiments fully support these relations for different epoxides and polyimides. ${ }^{10,12,13} \mathrm{At}$ low $T_{\text {cure }}$ the values of $T_{\mathrm{g}}$ change approximately linearly up to $T_{\mathrm{g}}^{\max }$.

The given results have pronounced technical importance especially for the synthesis of high temperature polymers and composites based on them.

The relations 1 and 2 show that it is impossible to prepare a polymer with high $T_{\mathrm{g}}$ at a low $T_{\text {cure }}$, at least in polyaddition or polycondensation reactions.

This conclusion is practically important for the preparation of polymers with $T_{\mathrm{g}}$ higher than their degradation temperature. Direct measurement of the $T_{\mathrm{g}}$ of those polymers is practically unreliable and usually $T_{\mathrm{g}}$ for them is estimated by the method of increments. ${ }^{14}$ The real value of $T_{\mathrm{g}}$ for those polymers should be found from the relation

$$
T_{\mathrm{g}} \approx T_{\text {cure }}
$$

and calculations of $T_{\mathrm{g}}$ from increments may give wrong results. In order to prepare really high temperature glassy polymer (or polymer matrix in composite) by using the abovementioned chemical processes one should heat a cured polymer up to the temperature of its real use.

The topic of chemical vitrification was dis-

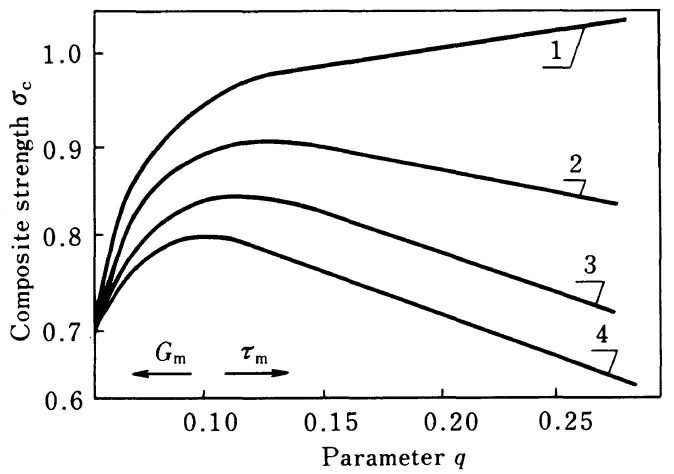

Figure 4. Composite strength $\sigma_{\mathrm{c}}$ (computations) as a function of $q .{ }^{16}$ Curves $1-4$ are differed by defect dimensions (the number $N$ of broken fibers in the technological defect in FRP). 1, N=0 (defectless composite structure); $2, N=1 ; 3, N=4 ; 4, N=20 . G_{\mathrm{m}}, \tau_{\mathrm{m}}$ : shear modulus and shear yield stress of matrix.

cussed only for an epoxy cure process. However, it looks that this phenomenon is a general one for different chemistries and processes. Chemical vitrification should be considered as an important scientific and practical aspect of polymer and advanced composites material science.

\section{PLASTIC DEFORMATION OF GLASSY POLYMERS}

Good structural material should be ductile and not brittle. One of the main advantages of metals is their high ability to undergo plastic deformation and any new nonmetallic structural material should have an ability to undergo plastic deformation comparable with that of, at least, bad metals. That is a great desire of engineers. The historical way to create structural glassy materials was, to a large extent, the struggle against brittleness. Quite fortunately polymeric glasses are not intrinsically brittle. ${ }^{15}$

The problem of organic polymer matrix ductility in advanced composites has some new aspects. Quite often chemists try to synthesize a matrix polymer with high rigidity and high strength. However the increase of matrix strength and rigidity is not always followed by the growth of FRP strength. The exam- 
ple, given in Figure 4, is adopted from the literature. ${ }^{16}$ In this work the computations were performed for a model composite (unidirectional) with brittle high moduli-high strength fibers and a plastic (mechanically) matrix, and this model composite was stressed by tensile load along the reinforcing fibers.

Figure 4 shows, as the result of computations, the change of FRP strength $\sigma_{\mathrm{c}}$ with the parameter $q$ which depends on the mechanical properties of a matrix $\left(\tau_{\mathrm{m}}\right)$ and composite $\left(E_{\mathrm{c}}, G_{\mathrm{c}}\right)$

$$
q=\frac{2 \tau_{\mathrm{m}}}{\pi \sigma} \sqrt{E_{\mathrm{c}} / G_{\mathrm{c}}}
$$

where $\tau_{\mathrm{m}}$ is the yield stress of matrix at shear; $\sigma$-acting tensile stress: $E_{\mathrm{c}}$ and $G_{\mathrm{c}}$-the Young and shear moduli of composite. The polymer matrix strength $\left(\tau_{\mathrm{m}}\right)$ increases in the direction of $q$ growth, and matrix rigidity $\left(G_{\mathrm{m}}\right)$ becomes smaller in this direction.

With the growth of matrix strength $\tau_{\mathrm{m}}$, the strength of the composite $\sigma_{c}$ systematically grows only for the ideal FRP structure (curve $1)$, that is the structure which has no mechanical technological defects (break of one or several reinforcing fibers in the initial material, for example). Unfortunately the preparation of FRP without technological defects is practically impossible. The situation becomes pronouncedly different in the case of an FRP with existing technological defects (curves 2-4). In this case stress concentration around defect increases with $q$ and $\tau_{\mathrm{m}}$.

At small $q$ (i.e., large $G_{\mathrm{m}}$ and small $\left.\tau_{\mathrm{m}}\right)-\sigma_{\mathrm{c}}$ is growing and comes to a maximum. From this point the FRP becomes extremely sensitive to defect size $N$ (see legend of Figure 4) composite strength $\sigma_{\mathrm{c}}$ is decreased with an increase of matrix strength $\tau_{\mathrm{m}}$. The bigger the defect the sharper the $\sigma_{\mathrm{c}}$ decrease is. At the maximum point in Figure 4 there is a change of the fracture mechanisms of FRP. At $q<q_{\max }$ the stress concentration around the defect is negligible and FRP fracture is occurring in a viscous (plastic) manner. At $q=q_{\max }$ the FRP sensitivity to the defect becomes very high and at $q>q_{\max }$ FRP fracture becomes brittle and happens through fast crack growth. Many experiments on composites could be explained in these terms. ${ }^{16}$ This important work shows that a good matrix for advanced composite, glass FRP at least, should have high values of $G_{\mathrm{m}}$ and low values of $\tau_{\mathrm{m}}$. It should be pointed out that shear yield stress of matrix in a bulk $\tau_{\mathrm{m}}^{\mathrm{b}}$ becomes twice higher in the composite due to matrix deformation constraints. ${ }^{16}$

Immediately the question arises: can we, polymer chemists, synthesize today a glassy polymer with those characteristics? Analysis of the literature data on mechanical properties of glassy polymers brings great doubts that we can. It is evident now that the Young (and shear) moduli of isotropic glassy polymers practically do not depend on polymer chemical composition at least for known polymers. That reflects a narrow distribution of glassy polymers packing density and the role of Van der Waals interactions in their packing. ${ }^{17}$ Experimental shear yield stresses of glassy polymers at very low temperatures reach the magnitudes of theoretical Frenkel values $\tau_{\mathrm{y}}^{\text {th }},{ }^{18}$

$$
\tau_{\mathrm{y}}^{\mathrm{th}} \approx 10^{-1} G_{\mathrm{m}}^{\mathrm{th}}
$$

Quite fortunately at higher temperatures the experimental shear yield stress of polymeric glasses becomes much lower ${ }^{19}$

$$
\tau_{\mathrm{y}}^{\exp }<10^{-2} G_{\mathrm{m}}^{\mathrm{th}}
$$

However that is still higher than that of metals $\left(\tau_{\mathrm{y}}^{\mathrm{exp}} \approx 10^{-3}-10^{-5} G_{\mathrm{m}}^{\mathrm{th}}\right)^{20}$ and the preparation of polymeric glasses with the ratio $\tau_{y}^{\exp } /$ $G_{\mathrm{m}}^{\mathrm{th}} \sim 10^{-3}$ or even smaller, that is the same as in metals, is a challenging problem for polymer chemistry.

So, the situation, perhaps, may be summarized in the following way: polymer material science, at least for structural materials including advanced composites, must meet new demands. For the new generation of advanced composites we should create a new family of glassy polymers with high enough $G_{\mathrm{m}}$ 
and the ratio between shear yield stress and shear modulus on the order of $10^{-3}$, that is with mechanical properties which do not exist for the known glassy polymers. It is not clear now if the problem is soluble or not. However a next generation of new structural materials based on glassy polymers, perhaps, should overcome this barrier.

Thus the polymer community confronts a new complicated problem.

Why the metals are so plastic? The answer is very clear, there are special types of defectsthe dislocations, which are easily generated and grew under mechanical stresses in metals and dislocation dynamics is responsible for this desirable metallic property. ${ }^{20}$

One cannot really change the isotropic glassy polymer modulus. The only way to improve polymer mechanical properties in a desirable manner is enhancing of their ability to plastic flow. What is the way of doing this? This question is fundamental in the polymer field and glassy state of matter in general terms. If some specific defects in polymer bulk (like dislocations in metals) with high ability to plastic flow may exist it opens some hopes for the future.

Nevertheless the mechanism of plastic deformation of polymeric glasses is a subject of large uncertainties and controversies. ${ }^{21}$

In this paper some new results in the field ${ }^{22}$ will be considered. Perhaps, it will give some new enlightenment concerning this problem. We measured the linear dimension recoverability on heating of plastically deformed samples of several glassy polymers: commercial grade atactic polystyrene (a-PS) and aPMMA, polycarbonate, PVC and a densely crosslinked epoxy-amine network (EAN) DGER- $m$-PhDA $(1: 1)$ (see previous part). The samples were deformed by unidirectional compression, tension or shear up to the desirable value of deformation $\varepsilon_{\text {def }}$ and unloaded immediately or in the regime of stress relaxation $\left(\varepsilon_{\mathrm{def}}=\mathrm{const}: \sigma(t) \rightarrow 0\right)$. After complete unloading glassy samples have stored

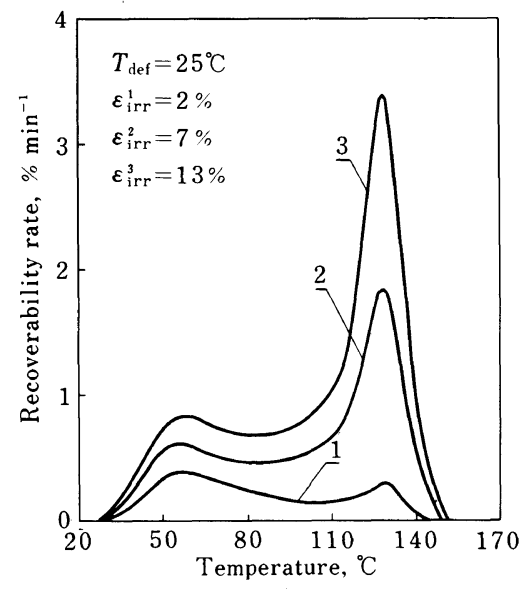

Figure 5. Dimension recoverability curves of plastically deformed samples of EAN on heating. $T_{\text {def }}=25^{\circ} \mathrm{C}$. $\varepsilon_{\text {irr }}: 1,2 \% ; 2,7 \% ; 3,13 \%$. Heating rate $=5^{\circ} \mathrm{C} \mathrm{min}^{-1}$.

some irreversible deformation $\left(\varepsilon_{\mathrm{irr}}\right)$, the value of which was the function of $\varepsilon_{\text {def }}$ and unloading regime.

The deformed samples were heated linearly $(\mathrm{d} T / \mathrm{d} t=\mathrm{const})$ and the dimension change $\varepsilon$ and its first derivative $\dot{\varepsilon}$ were registrated by using a TMA-2 Perkin-Elmer apparatus (for details, see ref 22). By performing these measurements we found several interesting results.

- All irreversibly deformed glassy polymers recovered completely on heating $\left(\varepsilon_{\mathrm{irr}} \rightarrow 0\right)$. Their recoverability happens in two remarkably separate steps (Figure 5). This distinguishes the glassy state of the polymers from their rubbery state.

- The position of the low temperature dimension recoverability step and its intensity depends on the deformation conditions. Its temperature position depends on $T_{\text {def }}$. The intensity of the peak grows with lowering $T_{\text {def }}$ and at sufficiently low $T_{\text {def }}$ practically all $\varepsilon_{\text {irr }}$ recovers through the low temperature dimension recoverability step (see Figure 6).

This result is very important. It shows that irreversible deformation in glassy polymers is able to recover at temperatures much lower 


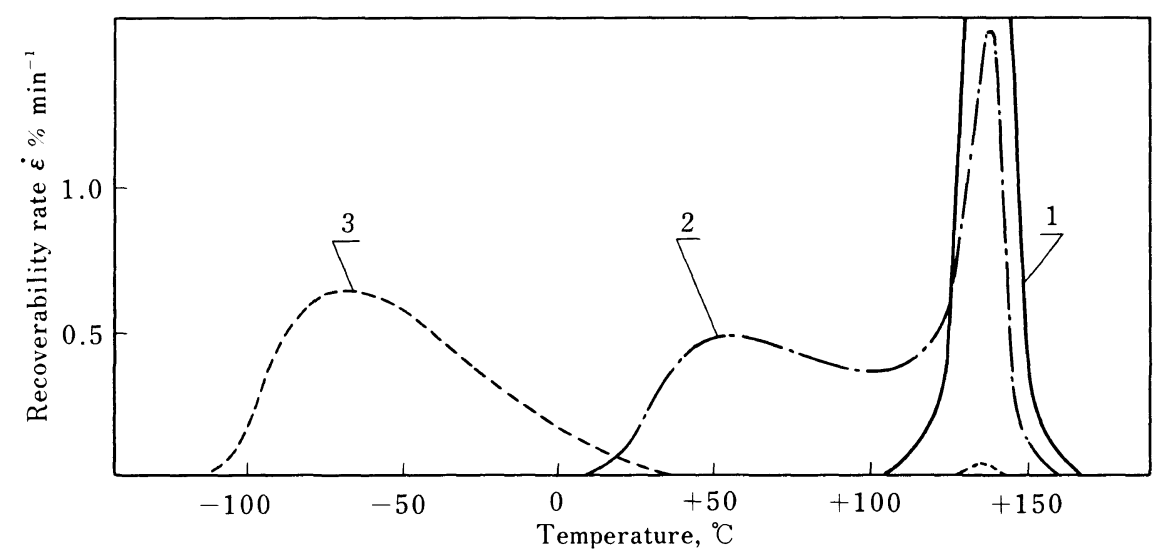

Figure 6. Dimension recoverability curves of plastically deformed samples of EAN. $1, \varepsilon_{\mathrm{irr}}=15 \%, T_{\text {def }}=$ $150^{\circ} \mathrm{C}$ with following cooling to room temperature at $\varepsilon_{\text {irr }}=$ const. (stress relaxation regime). $2, T_{\text {def }}=20^{\circ} \mathrm{C}$; $\varepsilon_{\text {irr }}=9.0 \%$; fast unloading. $3, T_{\text {def }}=-90^{\circ} \mathrm{C} ; \varepsilon_{\text {irr }}=8.5 \%$; fast unloading.

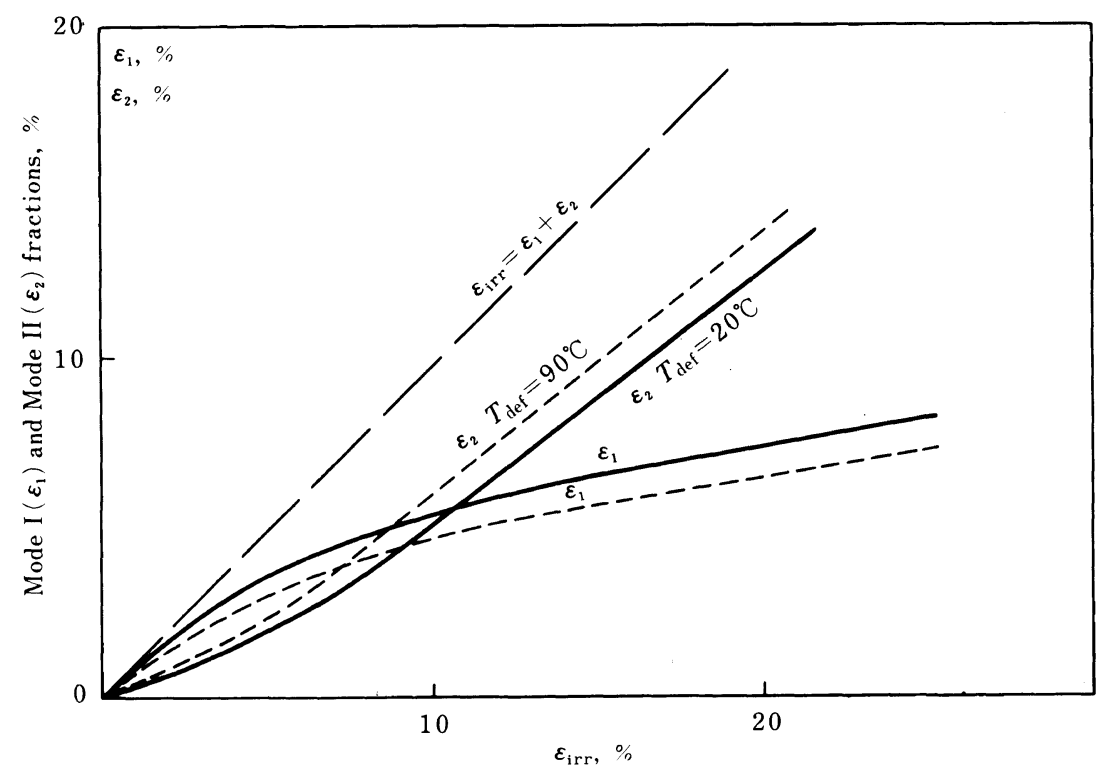

Figure 7. Fractions of Mode I and Mode II dimension recoverability of plastically deformed samples of EAN at different $\varepsilon_{\text {irr }}$, solid lines, $T_{\text {def }}=20^{\circ} \mathrm{C}$; dotted lines, $T_{\text {def }}=90^{\circ} \mathrm{C}$.

than $T_{\mathrm{g}}$. In other words it means that the activation energy $U$ for the plastic deformation recoverability process may be quite small

$$
U \sim k T_{\text {def }} \quad \text { with } \quad T_{\text {def }} \ll T_{\mathrm{g}}
$$

The areas under the recoverability peaks in Figures 5 and 6 give the fractions of total $\varepsilon_{\text {irr }}$, which recover through the Mode I (low tem- perature) and Mode II (at $T_{\mathrm{g}}$ ) recoverability steps.

These fractions are shown in Figure 7 for different $\varepsilon_{\text {irr }}$ of EAN.

Figure 7 shows that in the deformed glassy polymer on heating there exist two different and probably independent processes of dimension recoverability, which, perhaps, reflects 


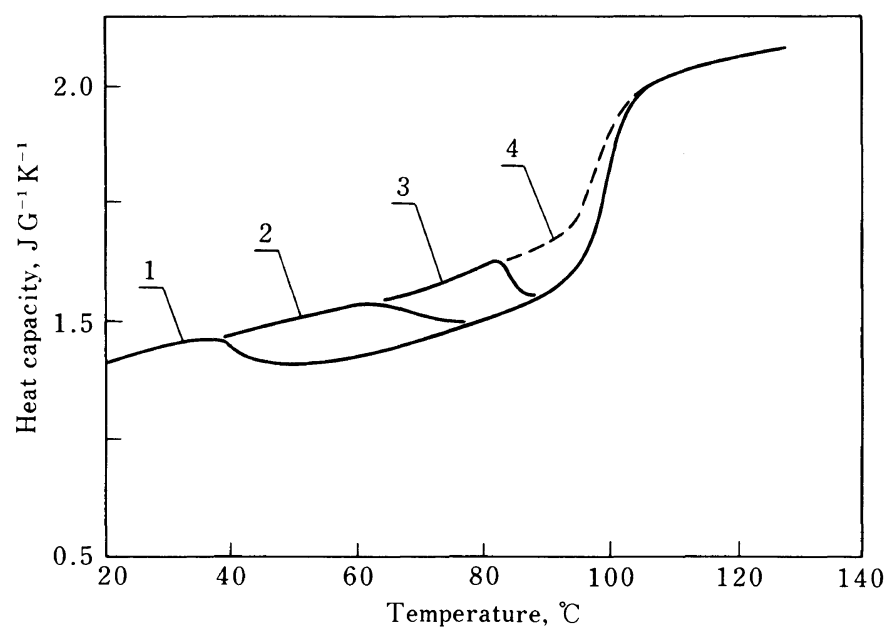

Figure 8. DSC traces of plastically deformed samples of a-PS at shear (samples were compressed up to $10 \mathrm{kbar}$ and sheared by $200^{\circ} \mathrm{C}$ degrees in Bridjmen envils). Heating rate $=20^{\circ} \mathrm{C} \mathrm{min}^{-1} .1, T_{\text {def }}=25^{\circ} \mathrm{C} ; 2$, $T_{\text {def }}=65^{\circ} \mathrm{C} ; 3, T_{\text {def }}=80^{\circ} \mathrm{C} ; 4$, second DSC run for samples $1,2,3$.

two different processes of plastic deformation. It should be pointed out that the same results have been obtained for all investigated polymers.

If the Mode II process is connected, as it broadly accepted, with conformation changes in polymeric chains, the Mode I process should differ from it. Figure 8 shows DSC traces for aPS deformed at different $T_{\text {def }}$. All DSC-curves of deformed samples show exothermic $C_{\mathrm{p}}$ anomalies. The temperature position of the anomalies follows $T_{\mathrm{def}}$. It is clear that the $C_{\mathrm{p}}$ anomalies and Mode I recoverability peaks are reflecting the same physical events-erasing of structural defects, which have appeared in polymer glass during preceding plastic deformation. The exothermic character of the $C_{\mathrm{p}^{-}}$ anomalies points out that conformational rearrangements are not dominating events in the creation of these deformation defects. These defects evidently carry mainly an excess of enthalpy but not an excess of enthropy.

Figure 7 shows that the plastic deformation of glassy polymers through the deformation of these kinds of energetic defects occurs mainly at the beginning of deformation process (small $\left.\varepsilon_{\text {irr }}\right)$ and the fraction of it increases at low $T_{\text {def }}$.
Under these conditions Mode I nonconformational type of plasticity has preferential character. However the process slows down at higher $\varepsilon_{\text {irr }}$ (Figure 7). The reason for this slowdown is not yet clear.

What kind of defects appears in polymeric glasses at low temperature under applied mechanical stress?

It is well known that at sufficiently low temperature in the solid state mass transport is possible only through slip events. Mass transport through holes (in Frenkel-Eyring sense) becomes impossible under these conditions. Simple estimates just show it.

The measurements of the deformation enthalpy $\Delta H$ by DSC and the heat of dissolution $\Delta Q$ of a-PS in toluene give the maximum values $4-5 \mathrm{~J} \mathrm{~g}^{-1}$. The value $\Delta Q$ increases to a plateau at $\varepsilon_{\text {irr }}=40-50 \%$.

The measurement of volume change $\Delta V$ in plastically deformed samples in comparison to annealed ones does not show any differences in the full temperature range $\Delta T=T_{\mathrm{g}}-T_{\text {def }}$. This means that the volume change of plastically deformed samples is lower than $4 \times 10^{-4}$ $\mathrm{cm}^{3} \mathrm{~g}^{-1}$ under our measurement conditions.

These results immediately give the value 


$$
\frac{\Delta H}{\Delta V} \approx 10^{10} \mathrm{~J} \mathrm{~m}^{-3}
$$

for this kind of deformation defects.

In contrast the nucleation of vacancies in organic crystals (ethyl alcohol, for example) gives the value $(1-2) \times 10^{9} \mathrm{~J} \mathrm{~m}^{-3}$, that is, one order of magnitude lower.

In solid-state physics the defects which may store a sufficient amount of energy with quite small volume change are well known. These defects are dislocations. ${ }^{20}$ The existence of dislocations in amorphous solids has been discussed for a long time. ${ }^{23}$ Now it is well accepted that dislocations may really exist in glassy substances. They should be of the Somigliana type and they are responsible for the plasticity of glasses..$^{3,21,23}$

Our results may be understood satisfactorily in this framework. The plastic deformation of glassy polymers may be considered as two independent processes. The low temperature process (Mode I) is a nucleation and growth of linear dislocation-type defects which are responsible for mass transport under mechanical stress. This kind of plastic deformation of glassy polymers is of a crystal-like type. Mode II deformation, recovering at $T_{\mathrm{g}}$, is the process which look like diffusional creep in crystals. ${ }^{21}$ Mass transport in this process is the diffusion of vacancy-like defects (holes) under the simultaneous action of mechanical stress and temperature. From Figure 7 it is evident that under any deformation conditions in polymer glasses Mode I plasticity develops as a first process and only in the vicinity of yield point does the Mode II process becomes noticeable.

Under working conditions the maximum deformation of structural plastics should be lower than $\varepsilon_{\mathrm{y}}$-the deformation at the yield point. This means that for structural plastics and advanced composites a dislocation-like deformation process is the most important one.

The dislocation dynamics in this case should be responsible for the level of the yield stress, deformation softenning, deformation rate sensitivity and, perhaps, for elementary fracture events. We do not know what kind of glassy polymer microstructure favors dislocation nucleation. We do not even know if these structural regions are of specific chemical origin or appear from packing defects.

However solutions in this field are necessary. Only desirable inclusion of the specific defects into the glassy polymer may help to create a rigid polymer with high plasticity, as is the case in metals behavior. This aim looks important in polymer material science.

\section{ENTHALPY AND VOLUME RELAXA- TIONS OF POLYMER GLASSES IN ISOTHERMAL AGING NEAR $T_{\mathrm{g}}$}

A serious problems in the physical chemistry of high polymers and related materials is the change of their properties with time, that is physical aging.

There are many physical and chemical factors responsible for aging. However this paper will be concerned with only one aspect of polymeric glasses physical aging - the isothermal aging at temperatures close to $T_{\mathrm{g}}$.

Chemical reactions which happen in different polymers under these aging conditions are multiple and diverse. However, their understanding is on the sufficiently high level. ${ }^{24}$

The situation is different as it is related to physical processes of aging. Structural rearrangements in aged glassy polymers and their relation to changes of important technical properties such as dimensional, mechanical, electric and many others-have no reasonable physical interpretation. The situation in the field roughly may be characterized as follows.

- The processes of so called isothermal volume recovery have been investigated carefully and broadly (for glassy polymers). Kinetics of the $V$-recovery is characterized by four general features such as: relaxation nonlinearity (non- 


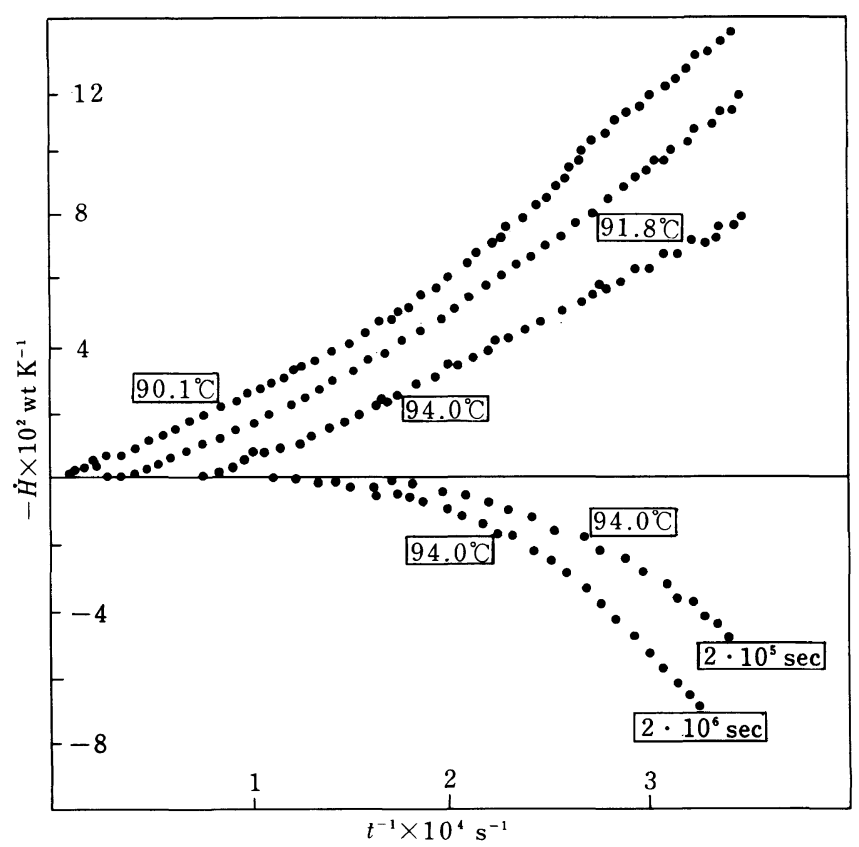

Figure 9. Enthalpy recovery rate $\dot{H}$ of a a-PS at different temperatures and at different (by values and signs) temperature jumps.

Upper part: Isothermal contraction at different $T_{\text {relax }}$ (shown in squares) after temperature jump from $T_{0}=$ $100^{\circ} \mathrm{C}$.

Lower part $:$ Isothermal expansion at $T_{\text {relax }}=94^{\circ} \mathrm{C}$ after temperature jump from $T_{0}=70^{\circ} \mathrm{C}$. Storage time at $T_{\text {relax }}=70^{\circ} \mathrm{C}: 2 \times 10^{5} \mathrm{~s}$ and $2 \times 10^{6} \mathrm{~s}$.

exponential relaxation kinetics), asymmetry (differential in $V$-recovery kinetics by approaching the same relaxation temperature from different temperature sides), hysteresis and memory effects. ${ }^{25,26}$

These features of $V$-recovery are general for the glassy state of matter and do not depend on the chemical nature of the investigated glass. They have been found for organic and inorganic glasses of high and low molecular weights and metallic glasses as well. ${ }^{26}$

-The description of these kinetic features of $V$-recovery are based on phenomenological multiparametric approach. This approach assumes the existence of a multiplicity of independent elementary relaxation processes during the structural recovery of the glassy state and the set of order parameters. There are the necessary conditions for the description of glassy state thermodynamics. ${ }^{24}$
- As a consequence of the multiparameter approach one should expect different relaxation rates for different physical properties under investigation such as a volume, enthalpy, electroconductivity, etc. Experiments with polymers have been usually interpreted in these terms. Nevertheless the careful measurement of the recovery kinetics of several different properties simultaneously are quite limited.

We have measured ${ }^{27}$ the simultaneous isothermal recovery with one sample of the enthalpy and volume for atactic polystyrene (aPS), PVC and PMMA using a Calvet-type calorimeter with a precision mercury dilatometer in the calorimeter cell. Measurements were performed by the method of temperature jumps. $^{25,27}$

Experimental results on enthalpy recovery are shown in Figure 9. It is clearly seen that the 


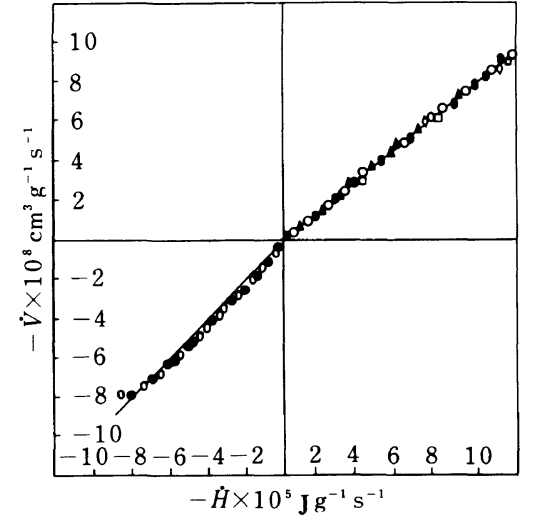

Figure 10. Correlation between the $\mathrm{d} V / \mathrm{d} t$ and $\mathrm{d} H / \mathrm{d} t$ for a-PS at different recovery conditions.

Upper right: Isothermal contraction after temperature jumps from $T_{0}=100^{\circ} \mathrm{C}$ to $T_{\text {relax }}: \bigcirc, 94^{\circ} \mathrm{C} ; \Delta, 92^{\circ} \mathrm{C} ; \boldsymbol{\Delta}$, $90^{\circ} \mathrm{C} ; \diamond, 84^{\circ} \mathrm{C} ; \square, 70^{\circ} \mathrm{C}$.

Lower left : Isothermal expansion at $T_{\text {relax }}=94^{\circ} \mathrm{C}$ after temperature jump from $T_{0}=70^{\circ} \mathrm{C}$. Storage time at $T_{\text {relax }}=94^{\circ} \mathrm{C}: \bigcirc, 5 \times 10^{5} \mathrm{~s} ; \bigcirc, 10^{5} \mathrm{~s}$.

enthalpy recovery kinetics show all the above mentioned features of $V$-recovery: hysteresis, asymmetry, memory and nonexponential character.

In Figure 10 is shown the relationship between $\dot{V}$ and $\dot{H}$ for all measurement conditions and for the full relaxation time interval. From Figure 10 it is evident that the rates of $V$ and $H$ relaxation show one-to-one correspondence in all circumstances, that is $\dot{V}=$ const. $\dot{H}$. This result definitely means that the relaxation rate of volume and enthalpy, two fundamental physical quantities, are controlled by the change of one structural parameter of the glass. Even for a limit of very long times (the extrapolation to the coordinate origin) there is no difference in the relaxation rates of volume and enthalpy. This conclusion is in some contradiction with the multiparameter concept of the glassy state relaxation kinetics in its usual sense.

But the problem still exists. In Figure 10 there is another important result. The slopes of the $\dot{V}$ vs. $\dot{H}$ lines extrapolated from both sides of coordinate origin are definitely different.
The slope of the lines $\partial H / \partial \mathrm{V}$ is an energy of new free volume formation or disappearance. (New free volume is formed in the sample when temperature jump $\Delta T=T_{\text {relax }}-T_{0}$ is positive and free volume is disappeared when $\Delta T$ is negative. $T_{\text {relax }}$ is the temperature of isothermal relaxation. $T_{0}$ is the temperature of sample storage before relaxation experiment. Negative $\Delta T$ relates to the experiments with sample contraction during relaxation measurement. Positive $\Delta T$ relates to the experiments with sample expansion).

If the free volume of formation disappearance is the process of vacancy formation the $\partial H / \partial V$ should be on the order of $10^{9} \mathrm{~J} \mathrm{~m}^{-3}$. The slope of the experimental line at negative temperature jump (upper right part of Figure 10) exactly corresponds to this value $\partial H=$ $(1-2) \times 10^{9} \mathrm{~J} \mathrm{~m}^{-3}$ and well corresponds to the $\Delta V=\Delta C_{\mathrm{p}}\left(T_{\mathrm{g}}\right) / \Delta \alpha\left(T_{\mathrm{g}}\right)$ ratio. The slope at positive temperature jump is higher by about $30 \%$ (lower left part of Figure 10 ). This values show that the main process of volume and enthalpy relaxation at temperatures close to $T_{\mathrm{g}}$ is the nucleation or disappearances of vacancies. However, the differences of the slopes for the negative temperature jump $\Delta T$ and the positive jump indicate that the relaxation of structural rearrangements in both cases are, perhaps, different.

We can not yet find a reasonable explanation for this result. Nevertheless we believe that a new type of asymmetry really exists. From the material science point of view this result means that samples of different thermal and mechanical prehistory may show complicated property changes during physical aging even if different macroscopic properties relax simultaneously.

\section{CONCLUSION}

The existing trend of broader use of glassy polymers in advanced materials creates many new unsolved scientific and technical problems which should be overcome by polymer scien- 
tists and engineers. However the main barrier for progress in the filed are the fundamentals of the structure-properties relationship for solid amorphous bodies.

The idea of ideal regular lattices lie at the basis of current concepts of solid state physics. The concept, at present, are definitely useless in the solution of glassy solid problems. Nevertheless it is important to remember that the disordered structures are spread out in nature much wider than regular ones.

One of the urgent problems of solid state physics and chemistry as well as material science today is overcoming this conceptual barrier. That is a great challenge for science.

\section{REFERENCES}

1. D. Adler, B. B. Schwartz, and M. C. Steele, Ed., "Physical Properties of Amorphous Materials," Plenum Press, New York/London, 1984.

2. K. K. Ngai and G. B. Wright, Ed., "Relaxation in Complex Systems," Symp. NRL, Washington DC 20375-5000, 1985.

3. J. J. Gilman and X. Y. Liuni, Ed., "Metallic Glasses," The American Society of Metals, Metals Park, Ohio, 1978.

4. R. Zallen, "Physics of Amorphous Solid," Elsvier, New York, 1982.

5. J. M. Ziman, "Models of Disorder," Cambridge University Press, New York/London, 1979.

6. E. A. Di Marsio, Ann. N. Y. Acad. Sci., 371, 1 (1981).

7. J. C. Seferis and L. Nicolais, Ed., "The Role of Polymeric Matrix in the Processing and Structural Properties of Composite Materials," Plenum Press, New York, 1983.

8. Y. K. Gillham, "Curing," in "Encyclopedia on Polymer Science and Engineering," Vol. 4, John Wiley \& Sons, New York, N. Y., 1986, pp 519-524.
9. J. K. Gillham, "Development in Polymer Characterization," Vol. 3, J. V. Dawkins, Ed., Applied Science, London, 1982, pp 159-227.

10. E. F. Oleinik, "Advance in Polymer Science," 80, Springer-Verlag, Berlin, 1986, pp 49-99.

11. J. Lubin, Ed., "Handbook of Composites," VNR Co., New York, N. Y., 1982.

12. E. F. Oleinik, O. B. Salamatina, E. L. Akopyan, S. N. Rudnev, and S. I. Nazarenko, Khimich. Phyzika (SSSR), 3, 885 (1984).

13. Y. B. Enns and J. K. Gillham, J. Appl. Polym. Sci., 28, 2831 (1983).

14. D. W. van Krevelen, "Properties of Polymers," Elsevier Science Publishing, Co., New York, N. Y., 1972.

15. A. Kelly, "Strong Solids," Clarendon Press, Oxford, 1973.

16. S. L. Bajenov and A. A. Berlin, Dokl. Acad. Nauk $S S S R, 283,1386$ (1986).

17. R. Haward, "Physics of Glassy Polymers," R. Haward, Ed., Applied Science Publishers, London, 1973.

18. Ja. Frenkel, Z. Phys., 37, 572 (1926).

19. B. Escaig, Polym. Eng. Sci., 24, 737 (1984).

20. J. Friedel, "Dislocation," Pergamon Press, Oxford, 1964.

21. C. Gsell and B. Escaig, Ed., "Plastic Deformation of Amorphous and Semicristalline Materials," Physique.Publ., Les Ulis, 1982.

22. E. F. Oleinik, S. N. Rudnev, O. B. Salamatina, S. I. Nazarenko, and G. A. Grigoryan, Dokl. Acad. Nauk SSSR, 286, 135 (1986).

23. J. J. Gillman, "Dislocation Dynamics," A. R. Rosefeld, Ed., McGraw-Hill, New York, N. Y., 1968.

24. Yu. V. Moiseev and G. E. Zaikov, "Chemical Resistance of Polymers in Reactive Media," Plenum Press, New York, N. Y., 1986, p 565.

25. A. J. Kovacs, Adv. Polym. Sci., 3, 394 (1963).

26. V. V. Bulatov, A. A. Gusev, and E. F. Oleinik, Macromol. Chem. Suppl., 6, 305 (1984).

27. A. A. Gusev, S. I. Beshenko, and E. F. Oleinik, Dokl. Acad. Nauk. SSSR, 288, 1143 (1986). 